\title{
Charged $\rho$ Meson Condensate in Neutron Stars within RMF Models
}

\author{
Konstantin A. Maslov 1,2,*, Evgeni E. Kolomeitsev ${ }^{2,3}$ (i) and Dmitry N. Voskresensky 1,2 \\ 1 Department of Theoretical Nuclear Physics, National Research Nuclear University (MEPhI), \\ Kashirskoe sh. 31, Moscow 115409, Russia; d.voskresen@gmail.com \\ 2 Joint Institute for Nuclear Research, Joliot-Curie 6, Dubna 141980, Russia; E.Kolomeitsev@gsi.de \\ 3 Department of Physics, Matej Bel University, Tajovského 40, 97401 Banská Bystrica, Slovakia \\ * Correspondence: maslov@theor.mephi.ru
}

Received: 30 November 2017; Accepted: 12 December 2017; Published: 26 December 2017

\begin{abstract}
Knowledge of the equation of state (EoS) of cold and dense baryonic matter is essential for the description of properties of neutron stars (NSs). With an increase of the density, new baryon species can appear in NS matter, as well as various meson condensates. In previous works, we developed relativistic mean-field (RMF) models with hyperons and $\Delta$-isobars, which passed the majority of known experimental constraints, including the existence of a $2 M_{\odot}$ neutron star. In this contribution, we present results of the inclusion of $\rho^{-}$-meson condensation into these models. We have shown that, in one class of the models (so-called KVOR-based models, in which the additional stiffening procedure is introduced in the isoscalar sector), the condensation gives only a small contribution to the EoS. In another class of the models (MKVOR-based models with additional stiffening in isovector sector), the condensation can lead to a first-order phase transition and a substantial decrease of the NS mass. Nevertheless, in all resulting models, the condensation does not spoil the description of the experimental constraints.
\end{abstract}

Keywords: neutron stars; equation of state; $\rho$ meson condensation; maximum mass; $\Delta$ resonances

\section{Introduction}

The equation of state (EoS) of strongly interacting hadronic matter is an essential input for describing the properties of neutron stars (NSs). A viable EoS has to fulfill various constraints following from both astrophysical observations and nuclear experimental data [1]. The discovery of the most massive pulsar with the mass $M=2.01 \pm 0.04 M_{\odot}$ [2] put a severe constraint, ruling out many soft EsoS. Currently, one of the most challenging tasks for phenomenological models of the EoS is to pass simultaneously the maximum NS mass constraint, requiring the EoS to be stiff, and the so-called flow constraint [3] coming from the analysis of flows in heavy ion collisions, which requires a soft EoS. This is hard to achieve within traditional models.

Relativistic mean-field (RMF) framework is a convenient and successful tool for constructing the nuclear equation of state. With an increase of the density hyperons and $\Delta$ isobars can appear in the NS matter [4]. In most of known model, this leads to a decrease of the maximum NS mass to unrealistic values. Another reason of the EoS softening is the possible appearance of the charged $\rho$-meson condensate in NS matter [5]. An RMF EoS can be made more flexible by introducing the dependence on the scalar field of the effective couplings and masses of all hadrons. In [6], we constructed new models within this approach, which fulfill the maximum NS mass constraint and the flow constraint simultaneously together with many other constraints, even with hyperons and $\Delta$ isobars included. In the current contribution, we demonstrate results of the inclusion of the charged $\rho$-meson condensate into our models. More details on the calculations can be found in [7]. 


\section{Description of the Model}

Our model was initially formulated in [8] and extended in $[6,7,9]$. Without the $\rho^{-}$meson condensation, the energy density of the model reads:

$$
\begin{aligned}
E\left[\left\{n_{b}\right\},\left\{n_{l}\right\}, f\right]= & \sum_{b} E_{\mathrm{kin}}\left(p_{\mathrm{F}, b}, m_{b} \Phi_{b}(f), s_{b}\right)+\sum_{l=e, \mu} E_{\mathrm{kin}}\left(p_{\mathrm{F}, l}, m_{l}, s_{l}\right)+\frac{m_{N}^{4} f^{2}}{2 C_{\sigma}^{2}} \eta_{\sigma}(f) \\
+ & \frac{1}{2 m_{N}^{2}}\left[\frac{C_{\omega}^{2} n_{V}^{2}}{\eta_{\omega}(f)}+\frac{C_{\rho}^{2} n_{I}^{2}}{\eta_{\rho}(f)}+\frac{C_{\phi}^{2} n_{S}^{2}}{\eta_{\phi}(f)}\right], \\
& E_{\mathrm{kin}}\left(p_{\mathrm{F}}, m, s\right)=(2 s+1) \int_{0}^{p_{\mathrm{F}}} \frac{p^{2} d p}{2 \pi^{2}} \sqrt{p^{2}+m^{2}}, \\
n_{V}= & \sum_{b} x_{\omega b} n_{b}, \quad n_{I}=\sum_{b} x_{\rho b} t_{3 b} n_{b}, \quad n_{S}=\sum_{H} x_{\phi H} n_{H} .
\end{aligned}
$$

Here, we introduced the dimensionless scalar field $f=g_{\sigma N} \chi_{\sigma N}(\sigma) \sigma / m_{N}$. The isospin projection of baryon $b$ is $t_{3 b}$, and $p_{\mathrm{F}, j}=\left(6 \pi^{2} n_{j} /\left(2 s_{j}+1\right)\right)^{1 / 3}$ denotes the Fermi momentum of a fermion $j$, with $s_{j}$ and $n_{j}$ being the spin and density of a species $j$, respectively, $j=(b, l), l$ labels leptons. In the infinite hadronic matter without meson condensates, the energy density depends only on the ratios of the meson coupling constants, masses and their corresponding scaling functions, namely

$$
\begin{gathered}
C_{M}=\frac{g_{M N} m_{N}}{m_{M}}, M=\sigma, \omega, \rho, \quad C_{\phi}=\frac{g_{\omega N} m_{N}}{m_{\phi}}, \\
\eta_{\omega}(f)=\frac{\Phi_{\omega}^{2}(f)}{\chi_{\omega N}^{2}(f)}, \eta_{\rho}(f)=\frac{\Phi_{\rho}^{2}(f)}{\chi_{\rho N}^{2}(f)}, \eta_{\phi}(f)=\frac{\Phi_{\phi}^{2}(f)}{\chi_{\phi H}^{2}(f)}, \eta_{\sigma}(f)=\frac{\Phi_{\sigma}^{2}[\sigma(f)]}{\chi_{\sigma N}^{2}[\sigma(f)]}+\frac{2 C_{\sigma}^{2}}{m_{N}^{4} f^{2}} U[\sigma(f)] .
\end{gathered}
$$

Here, the the self-interaction potential $U(\sigma)$ entering the Lagrangian of the model is hidden into the scaling function $\eta_{\sigma}(f)$. The coupling constant ratios for various baryons are defined as $x_{M b}=g_{M b} / g_{M N}, x_{\phi H}=g_{\phi H} / g_{\omega N}$. We refer the reader to [6,9] for explicit expressions for the scaling functions $\eta_{m}(f)$ and values of the parameters for all our models. Below, we use $\chi_{M b}=\chi_{M N}, \chi_{\phi H}=1$.

The baryon coupling ratios with vector mesons $x_{\omega B}$ and $x_{\rho B}$ are determined by the quark SU(6) symmetry. The baryon coupling ratios with the scalar field $x_{\sigma B}$ follow from the potentials

$$
U_{B}\left(n_{0}\right)=C_{\omega}^{2} m_{N}^{-2} x_{\omega B} n_{0} / \eta_{\omega}\left(f\left(n_{0}\right)\right)-x_{\sigma B}\left(m_{N}+m_{N}^{*}\left(n_{0}\right)\right)
$$

in the isospin-symmetric matter (ISM) at the saturation density $n=n_{0}$. The $\Delta$ potential $U_{\Delta}\left(n_{0}\right) \equiv U_{\Delta}$ is a subject of large uncertainties. Here we assume $U_{\Delta}=-50 \mathrm{MeV}$, following from the most realistic estimate [10]. The values of the parameters for all included baryon species are given in [6].

\section{Charged $\rho$ Condensate}

The $\rho$ meson field is described by the following Lagrangian $[5,8]$

$$
\begin{aligned}
\mathcal{L}_{\rho} & =-\frac{1}{4} \vec{R}_{\mu v} \vec{R}^{\mu v}+\frac{1}{2} m_{\rho}^{2} \Phi_{\rho}^{2} \vec{\rho}_{\mu} \vec{\rho}^{\mu}-\sum_{b} g_{\rho b} \chi_{\rho b} \vec{\rho}_{\mu} \vec{j}_{I, b}^{\mu}, \quad \vec{j}_{I, b}^{\mu}=\bar{\psi}_{b} \gamma^{\mu} \vec{t}_{b} \psi_{b}, \\
\vec{R}_{\mu v} & =\partial_{\mu} \vec{\rho}_{v}-\partial_{\nu} \vec{\rho}_{\mu}+g_{\rho}^{\prime} \chi_{\rho}^{\prime}(f)\left[\vec{\rho}_{\mu} \times \vec{\rho}_{\nu}\right]+\mu_{\mathrm{ch}, \rho} \delta_{\nu 0}\left[\vec{n}_{3} \times \vec{\rho}_{\mu}\right]-\mu_{\mathrm{ch}, \rho} \delta_{\mu 0}\left[\vec{n}_{3} \times \vec{\rho}_{\nu}\right],
\end{aligned}
$$

where the chemical potential $\mu_{\mathrm{ch}, \rho}$ is introduced for charged mesons and $\left(\vec{n}_{3}\right)^{a}=\delta^{a 3}$ is the unit vector in the isospin space. We treat $\rho$ meson as a gauge boson of a hidden local symmetry and introduce the non-Abelian coupling constant $g_{\rho}^{\prime}$ and its scaling function $\chi_{\rho}^{\prime}(f)$. Hidden local symmetry requires that $g_{\rho}^{\prime}=g_{\rho N}$, which we use here, and for simplicity we consider $\chi_{\rho}^{\prime}(f)=1$. In the standard ansatz for the $\rho$-meson mean fields only the $\rho_{0}^{(3)}$ component is non-zero. This ansatz was used in obtaining 
Equation (3). The charged $\rho^{-}$meson condensation can be introduced by using the new ansatz for the $\rho$ meson field:

$$
\rho_{0}^{(3)} \neq 0, \quad \rho_{i}^{ \pm}=\left(\rho_{i}^{(1)}+\rho_{i}^{(2)}\right) / \sqrt{2} \neq 0, \quad i=1,2,3
$$

It can be shown that within this ansatz the minimum of energy is realized if the condition

$$
\rho_{i}^{(+)} \rho_{j}^{(-)}-\rho_{i}^{(-)} \rho_{j}^{(+)}=0
$$

is fulfilled. This is equivalent to the ratio $\rho_{i}^{(+)} / \rho_{i}^{(-)}$being constant and independent of the spatial index $i$. Thus we can assume that $\rho_{i}^{(-)}=a_{i} \rho_{c}$ and $\rho_{i}^{(+)}=a_{i} \rho_{c}^{+}$, where we defined the complex amplitude of the charged $\rho$ meson field $\rho_{c}$, and $\vec{a}=\left\{a_{i}\right\}$ is the spatial unit vector. In such terms the thermodynamic potential can be minimized by two distinct solutions for the $\rho_{0}^{(3)}$ and $\rho_{c}$ fields. The first one is the traditional solution with only $\rho_{0}^{(3)}$ being non-zero. The second solution is

$$
\begin{gathered}
\rho_{0}^{(3)}=\frac{\mu_{\mathrm{ch}, \rho}-m_{\rho} \Phi_{\rho}}{g_{\rho} \chi_{\rho}^{\prime}}, \quad\left|\rho_{c}\right|^{2}=\frac{\left(-n_{I}-n_{\rho}\right) \theta\left(-n_{I}-n_{\rho}\right)}{2 m_{\rho} \eta_{\rho}^{1 / 2} \chi_{\rho}^{\prime}}, \\
n_{\rho}=\frac{m_{N}^{2} \eta_{\rho}^{1 / 2} \Phi_{\rho}}{C_{\rho}^{2} \chi_{\rho}^{\prime}}\left(m_{\rho} \Phi_{\rho}-\mu_{\mathrm{ch}, \rho}\right) .
\end{gathered}
$$

The electric charge density of $\rho^{-}$is $n_{\mathrm{ch}, \rho}=-2 m_{\rho} \Phi_{\rho}(f)\left|\rho_{c}\right|^{2}<0$. The $\rho^{-}$meson condensate gives the following contribution to the energy density:

$$
\Delta E_{\mathrm{ch}, \rho}\left[\left\{n_{b}\right\} ; f\right]=-\frac{C_{\rho}^{2}}{2 m_{N}^{2} \eta_{\rho}}\left(n_{I}+n_{\rho}\right)^{2} \theta\left(-n_{I}-n_{\rho}\right)-\mu_{\mathrm{ch}, \rho} n_{\mathrm{ch}, \rho},
$$

where $\theta\left(-n_{I}-n_{\rho}\right)=1$ for $n_{I}+n_{\rho}<0$ and zero otherwise. In the presence of the condensate, the charge neutrality condition is modified to be $\sum_{b} Q_{b} n_{b}-n_{e}-n_{\mu}+n_{\mathrm{ch}, \rho}=0$. In the beta-equilibrium matter (BEM) of a NS the chemical potentials are related through conditions $\mu_{e}=\mu_{\mu}=\mu_{\mathrm{ch}, \rho}$, $\mu_{b}=\mu_{n}-Q_{b} \mu_{l}$. All equations are solved self-consistently with the equation of motion for the scalar field $\partial\left(E+\mu_{\mathrm{ch}, \rho} n_{\mathrm{ch}, \rho}\right) / \partial f=0$. Once the equilibrium concentrations are obtained, the pressure can be evaluated as $P=\sum_{j=b, l,\{\mathrm{ch}, \rho\}} \mu_{j} n_{j}-E$.

\section{Numerical Results}

In [7], we considered the MKVOR* and KVORcut03 model. We have shown that, in the KVORcut03 model, the $\rho^{-}$condensate does not appear in the most realistic case with the hyperons and/or $\Delta s$ taken into account. Thus, in this contribution, we focus on results for the MKVOR* model. Below, we present the results of the inclusion of the $\rho^{-}$condensate into the MKVOR* model with the universal mass scaling and check the sensitivity of the results to varying the scaling functions $\Phi_{\rho}(f)$ and $\eta_{\rho}(f)$. In the following, we denote the MKVOR* model with hyperons and $\Delta$ s included as $\mathrm{MKVOR}^{*} \mathrm{H} \Delta \phi$, and the inclusion of $\rho^{-}$condensate is denoted by the $\rho$ suffix.

\subsection{Inclusion of the Condensate}

In this section, we present the results for the MKVOR* model with the universal meson mass scaling $\Phi_{m}(f)=1-f, m=\{\sigma, \omega, \rho, \phi\}$, in accordance with [11-13]. In the left panel of Figure 1, we show the particle concentrations and the scalar field $f$ as functions of the total baryon densities. There exists a region of densities where several solutions for the particle fractions and the scalar field exist. This means that the system must prefer the branch of solutions with a lower energy. The density, 
for which such a transition from one branch of solutions to another happens, in our model, equals $n_{c}^{(I)}=2.81 n_{0}$. It is shown in Figure 1 by dotted vertical lines in the left and middle panels.
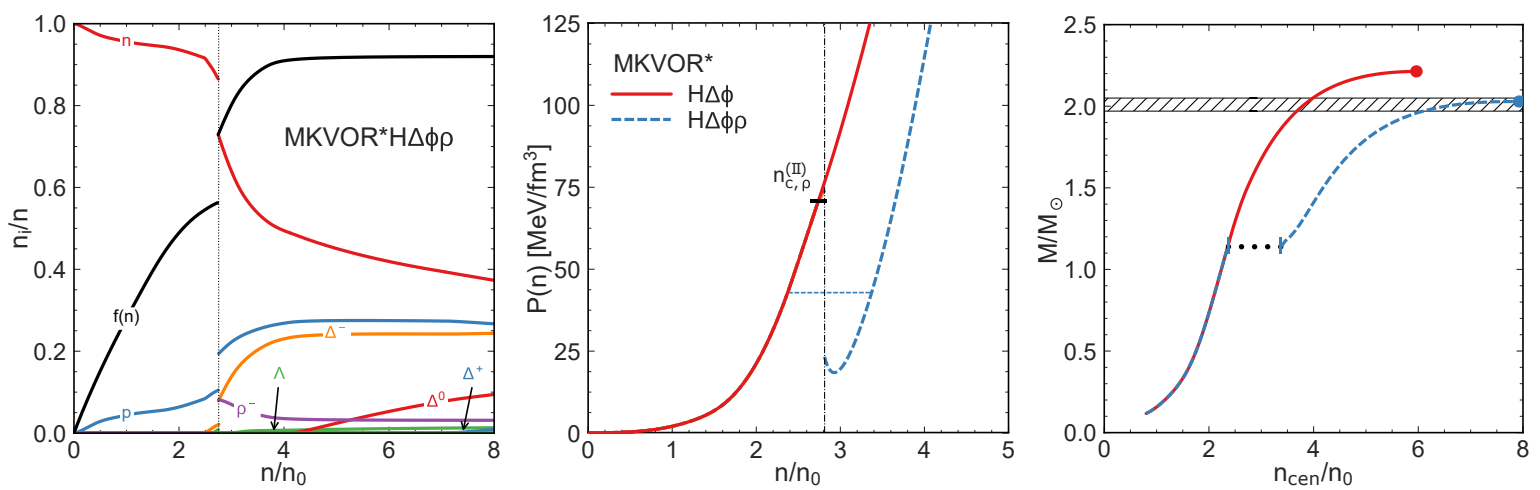

Figure 1. Left panel: Particle fractions together with the scalar field $f$ as functions of the total baryon density $n$ in the BEM for the MKVOR ${ }^{*} \mathrm{H} \triangle \phi \rho$ model. Only the energetically favorable regions of the solutions' branches are shown. Middle panel: The pressure as a function of the density for MKVOR ${ }^{*} H \Delta \phi$ and MKVOR ${ }^{*} H \Delta \phi \rho$ models. The short vertical dash shows the critical density for the 2nd order phase transition (PT). The vertical line in the left and middle panels denotes the density, at which the $\rho$-condensed phase becomes energetically more favorable than the normal one. Right panel: NS mass as a function of the central density for the same models as in the middle panel. Vertical dashes denote boundaries of the Maxwell construction region (shown by the dotted line), where no stable NS configurations exist. The horizontal stripe denotes the observational constraint $M_{\max } \geq(2.01 \pm 0.04) M_{\odot}$ [2] and large dots denote the maximum masses for the models.

The 1st order PT results in a van der Waals-like shape of the pressure, which, neglecting the possible pasta formation, should be replaced by a Maxwell construction, spanning over the densities $2.37 n_{0} \lesssim n \lesssim 3.37 n_{0}$. The resulting pressure as a function of the density is shown in the middle panel of Figure 1, where we compare the pressure with and without condensate for the MKVOR ${ }^{*} \mathrm{H} \Delta \phi$ model. The condensate appears not only on the new branch of solutions, but also at the old one with the critical density $n_{c}^{\text {(II) }} \simeq 2.74 n_{0}$, which is marked by a horizontal dash.

In the right panel of Figure 1, we show the NS mass as a function of the central density for $\mathrm{MKVOR}^{*} \mathrm{H} \Delta \phi$ with and without the inclusion of the condensate. We see that the $\rho^{-}$ condensation in this model results in a substantial decrease of the maximum NS mass from $2.21 \mathrm{M}_{\odot}$ to $2.03 M_{\odot}$. Nevertheless, even after such a reduction, the NS maximum mass still passes the observational constraint.

\subsection{Variation of the $\rho$-Meson Effective Mass}

A strong phase transition to the $\rho^{-}$-condensed state relies on the strong decrease of its effective mass. In this subsection, we study the effect of limiting the decrease of the $\rho$-meson effective mass using the following mass scaling function:

$$
\Phi_{\rho}(f)=\left\{\begin{array}{ll}
1-f & , f \leq f_{s} \\
\left(1-f_{s}\right)\left[1+\frac{\xi}{1+b_{\rho} \xi}\left(\frac{\xi}{2+b_{\rho}}-1\right)\right] & , f>f_{s}
\end{array} \quad \quad \xi=\frac{f-f_{s}}{1-f_{s}} .\right.
$$

This expression defines a one-parametric family of scaling functions, with a minimum value of the function $\Phi_{\rho, \min }$ as the parameter. For a given $\Phi_{\rho, \min }$, the value of $f_{s}$ is

$$
f_{s}=1-\Phi_{\rho, \min }-\delta \Phi_{\rho}
$$


where we introduce a constant offset $\delta \Phi_{\rho}=0.1$ allowing for a smooth transition at $f=f_{s}$. The parameter $b_{\rho}=\frac{\Phi_{\rho, \min }}{\delta \Phi_{\rho}}-1$ assures that $\Phi_{\rho}^{\prime}(f=1)=0$. Under the choice $\Phi_{\rho, \min }=0$, we will understand also $\delta \Phi=0$, which leads to $\Phi_{\rho}(f)=1-f$. In the left panel of Figure 2, we show the scaling function $\Phi_{\rho}(f)$ given by Equation (7) as a function of the scalar field $f$ for $\Phi_{\rho, \min }=0,0.3,0.5,0.7$, which we examine below. We see that for $\Phi_{\rho, \min }>0$ this function monotonously decreases, but asymptotically reaches $\Phi_{\rho}(1)=\Phi_{\rho \text {, min }}$. Thus this function $\Phi_{\rho}(f)$ is suitable for studying the effect of varying the decrease rate of the effective mass.
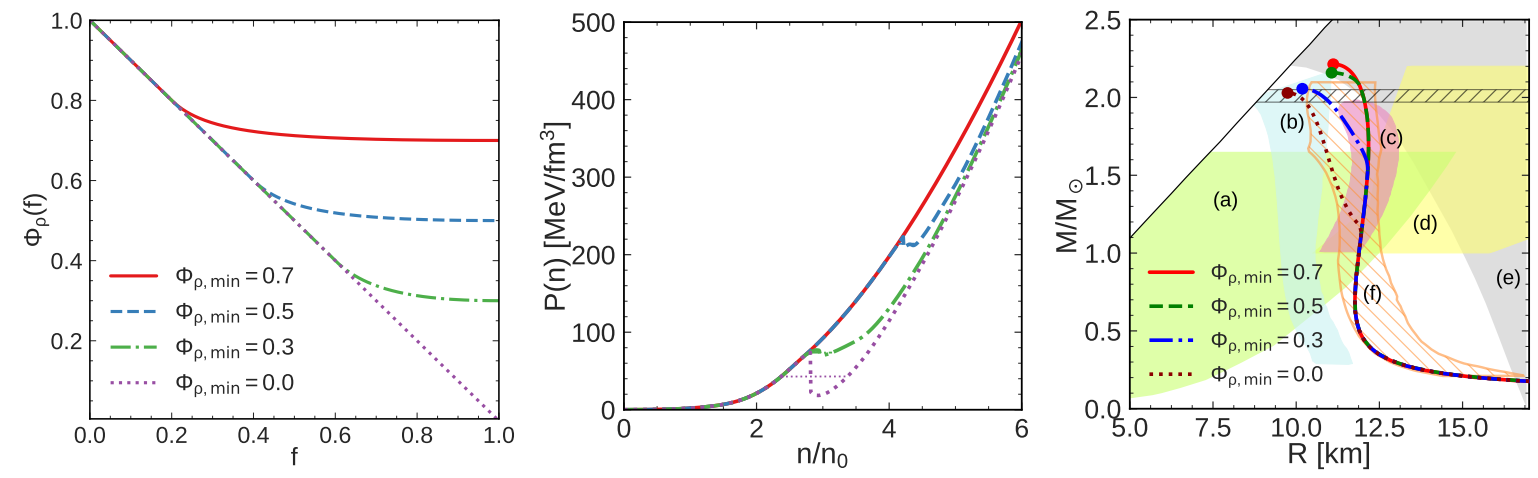

Figure 2. Left panel: the scaling function $\Phi_{\rho}(f)$ as a function of the scalar field $f$ defined by Equation (7) for $\Phi_{\rho, \min }=0,0.3,0.5,0.7$. Middle panel: the pressure as a function of the baryon density for the same values of $\Phi_{\rho, \min }$. Right panel: mass-radius curves for the same values of the parameters. For comparison, we show the emprical constraints: (a) [14]; (b) [15]; (c) [16]; (d) [17]; (e) [18]; and (f) [19-21]. The horizontal band shows the maximum NS mass constraint within the uncertainty range [2].

The resulting pressure as a function of the density is shown in the middle panel of Figure 2 . The 1 st order PT proves to be present for $\Phi_{\rho, \min }<0.7$. However, the corresponding critical density grows and the pressure loss decreases as we increase $\Phi_{\rho, \min }$. For $\Phi_{\rho, \min }=0.7$ no condensate appears in the model. The mass-radius curves for this models are shown in the right panel of Figure 2. The limiting of the decrease of $\Phi_{\rho}(f)$ leads to an increase of the maximum NS mass. The maximum NS mass $\Phi_{\rho \text {, min }}=0.3,0.5,0.7$ are $2.06,2.16$, and $2.21 M_{\odot}$, respectively, proven to be larger than $2.03 M_{\odot}$ in the case of $\Phi_{\rho}(f)=1-f$. Thus taming the $\rho$-meson effective mass decrease is an efficient way to control the effect of the $\rho^{-}$condensate.

\subsection{Variation of the $\eta_{\rho}(f)$}

Another reason for the large $\rho^{-}$condensate fraction is the particular shape of the $\eta_{\rho}(f)$ function in the $\mathrm{MKVOR}^{*}$ model. The sharp decrease in the $\eta_{\rho}(f)$ for $f \gtrsim 0.5$ is needed to quench the scalar field growth in the isospin-asymmetric matter, implementing the cut-mechanism of stiffening of the EoS [22]. However, as we shall see in this section, this choice of $\eta_{\rho}(f)$ corresponds to the maximum $\rho^{-}$ condensate in the NS matter. To show this, we investigate a set of scaling functions $\eta_{\rho}(f)$, smoothly changing their behavior from a sharp decrease at $f \gtrsim 0.5$ to a monotonous growth for all $f$.

The family of $\eta_{\rho}(f)$ we use here consists of functions $\eta_{\rho}^{(i)}(f), i=1 \ldots 17$ within three different analytic parameterizations labeled by an integer number. The details on the parameterizations can be found in [7]. The choice of $\eta_{\rho}^{(1)}$ corresponds to the original $\eta_{\rho}(f)$ of the MKVOR* model (tail 2). Dependence of $\eta_{\rho}^{(i)}(f), i=1 \ldots 17$ on the scalar field $f$ is shown in the left panel of Figure 3 . We see that with an increase of the model number gradually changes the $\eta_{\rho}^{(i)}$ behavior from sharply decreasing for $f \gtrsim 0.5$ to a monotonously growing function, thus switching off the cut-mechanism [22], which limits the growth of the scalar field.

In the right panel of Figure 3, we show the maximum NS mass as a function of the model index, while varying number of degrees of freedom included. Namely, we study the MKVOR*\{H $\phi, \mathrm{H} \Delta \phi$, 
$\mathrm{H} \phi \rho, \mathrm{H} \Delta \phi \rho\}$ models with $\Phi_{\rho}(f)=1-f$. For estimating the effect of limiting the decrease of the $\rho$-meson effective mass on these curves we consider also the MKVOR ${ }^{*} \mathrm{H} \Delta \phi \rho$ model with $\Phi_{\rho}$ given by Equation (7) with $\Phi_{\rho, \min }=0.5$. For the models without $\rho^{-}$condensate we see, that the choice $\eta_{\rho}=\eta_{\rho}^{(1)} \equiv \eta_{\rho}^{\mathrm{MKVOR}^{*}}$ maximizes the maximum NS mass, thus proving the efficiency of our "cut" mechanism of the EoS stiffening. In addition, one should notice that the difference between $\mathrm{H} \phi$ and $\mathrm{H} \Delta \phi$ curves is as well minimized by the $\eta_{\rho}^{(1)}$. It means that this choice of $\eta_{\rho}$ plays an important role in resolution of the " $\Delta$ puzzle" [23]. With an increase of the model number the impact of $\Delta s$ on the EoS grows, and for $i=17$ the maximum NS mass becomes $2.03 M_{\odot}$, marginally satisfying the maximum NS mass constraint.

The inclusion of the $\rho^{-}$condensate changes this tendency. The $\mathrm{H} \phi \rho$ curve monotonously increases with an increase of the model index and the choice $\eta_{\rho}=\eta_{\rho}^{(1)}$ minimized the maximum mass and maximizes the $\rho^{-}$condensate phase transition strength. A peculiar situation occurs if both $\Delta \mathrm{s}$ and $\rho^{-}$are included into the model. As one sees from the $\mathrm{H} \Delta \phi \rho$ curve in the right panel of Figure 3, the maximum NS mass is close to $2.03, M_{\odot}$ and almost independent on the model index. This happens because for low $i \rightarrow 1$ the softening comes from both the $\rho^{-}$condensate together with $\Delta \mathrm{s}$, and for large $i \rightarrow 17$ the $\rho^{-}$condensate disappears and the softening effect of $\Delta \mathrm{s}$ is increased, as was mentioned above. However, this independence of a maximum NS mass on the model number is accidental and holds only for $\Phi_{\rho}=1-f$. If we limit the decrease of the $\rho$-meson effective mass (see $\mathrm{H} \Delta \phi \rho$, $\Phi_{\rho, \min }=0.5$ curve in the right panel of Figure 3), such a degeneracy is removed.
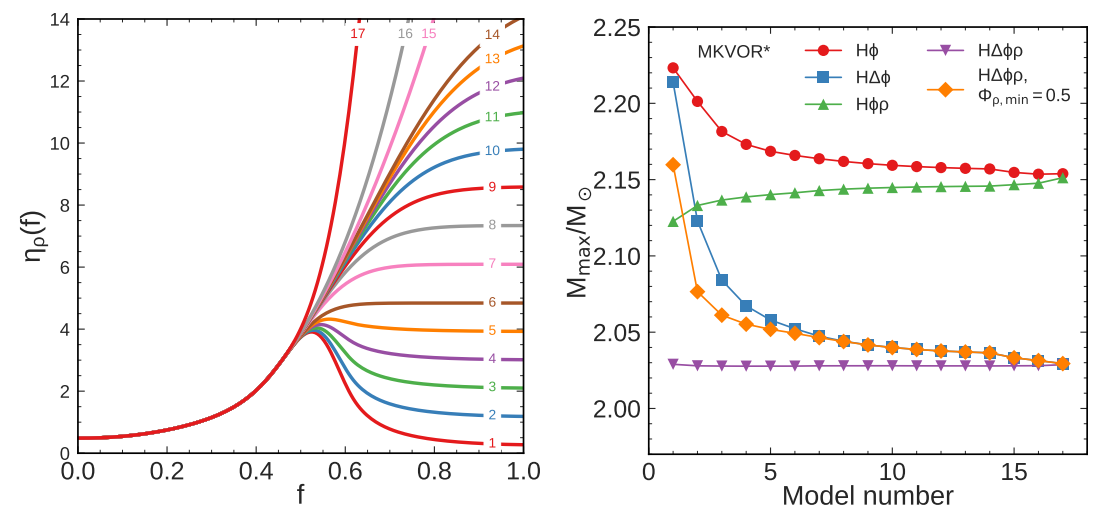

Figure 3. Left panel: Scaling functions $\eta_{\rho}^{(i)}$ for $i=1 \ldots 17$ as functions of the scalar field $f$. Right panel: Maximum NS mass as a function of the model number for $\mathrm{MKVOR}^{*}\{\mathrm{H} \phi, \mathrm{H} \Delta \phi, \mathrm{H} \phi \rho, \mathrm{H} \Delta \phi \rho\}$ models with $\Phi_{\rho}(f)=1-f$ and for MKVOR ${ }^{*} \mathrm{H} \Delta \phi \rho$ with $\Phi_{\rho}$ given by Equation (7) with $\Phi_{\rho, \min }=0.5$.

\section{Conclusions}

We studied a possibility of charged $\rho$-meson condensation in a realistic relativistic mean-field model MKVOR* with scaled hadron masses and couplings. The condensation proves to happen by a first-order phase transition and leads to a dramatic reduction of a predicted maximum NS mass, if one uses the universal scaling for masses of all mesons. Nevertheless, the NS maximum mass still passes the observational constraint. We have shown that limiting the decrease of the $\rho$-meson effective mass allow to reduce the effect of the phase transition on the EoS and increase the maximum neutron star mass. In addition, we demonstrated that our choice of the $\eta_{\rho}(f)$ scaling function maximizes the neutron star maximum mass in the case without $\rho^{-}$condensation. With the condensate included with the universal mass scaling, the maximum mass is almost independent of the choice of $\eta_{\rho}(f)$. This happens because if the scaling function is chosen to minimize the effect of the $\rho^{-}$condensate, the $\Delta$ abundance is increased, and vice versa. However, this effect proves to be accidental and does not manifest itself for a different $\rho$ meson mass scaling. 
Acknowledgments: The reported study was funded by the Russian Foundation for Basic Research (RFBR) according to the research project No 16-02-00023-A. The work was also supported by the Ministry of Education and Science of the Russian Federation within the state assignment, project No 3.6062.2017/BY, by the Slovak Grant No. VEGA-1/0469/15, and partially (Sections 4.2 and 4.3) by the Russian Science Foundation, Grant No. 17-12-01427. K.A.M. acknowledges the support by the grant of the Foundation for the Advancement of Theoretical Physics "BASIS". E.E.K acknowledges the support by the grant of the Plenipotentiary of the Slovak Government to JINR.

Author Contributions: All authors contributed equally to the formulation of the model, numerical analysis and writing of the paper.

Conflicts of Interest: The authors declare no conflict of interest.

\section{Abbreviations}

The following abbreviations are used in this manuscript:

$\begin{array}{ll}\text { EoS } & \text { Equation of state } \\ \text { NS } & \text { Neutron star } \\ \text { RMF } & \text { Relativistic mean-field } \\ \text { ISM } & \text { Isospin-symmetric matter } \\ \text { BEM } & \text { Beta-equilibrium matter } \\ \text { PT } & \text { Phase transition }\end{array}$

\section{References}

1. Klahn, T.; Klähn, T.; Blaschke, D.; Typel, S.; Van Dalen, E.N.E.; Faessler, A.; Fuchs, C.; Gaitanos, T.; Grigorian, A.; Ho, A.; et al. Constraints on the high-density nuclear equation of state from the phenomenology of compact stars and heavy-ion collisions. Phys. Rev. C 2006, 74, 035802.

2. Antoniadis, J.; Freire, P.C.; Wex, N.; Tauris, T.M.; Lynch, R.S.; van Kerkwijk, M.H.; Bassa, C.; Dhillon, V.S.; Hessels, J.W.T.; Kaspi, V.M.; et al. A Massive Pulsar in a Compact Relativistic Binary. Science 2013, $340,1233232$.

3. Danielewicz, P.; Lacey, R.; Lynch, W.G. Determination of the equation of state of dense matter. Science 2002, $298,1592-1596$.

4. Schaffner-Bielich, J. Hypernuclear Physics for Neutron Stars. Nucl. Phys. A 2008, 804, 309-321.

5. Voskresensky, D.N. On the possibility of the condensation of the charged rho meson field in dense isospin asymmetric baryon matter. Phys. Lett. B 1997, 392, 262-266.

6. Kolomeitsev, E.E.; Maslov, K.A.; Voskresensky, D.N. Delta isobars in relativistic mean-field models with sigma-scaled hadron masses and couplings. Nucl. Phys. A 2017, 961, 106-141.

7. Kolomeitsev, E.E.; Maslov, K.A.; Voskresensky, D.N. Charged $\rho$-meson condensation in neutron stars. Nucl. Phys. A 2018, 970, 291-315.

8. Kolomeitsev, E.E.; Voskresensky, D.N. Relativistic mean-field models with effective hadron masses and coupling constants, and rho- condensation. Nucl. Phys. A 2005, 759, 373-413.

9. Maslov, K.A.; Kolomeitsev, E.E.; Voskresensky, D.N. Relativistic Mean-Field Models with Scaled Hadron Masses and Couplings: Hyperons and Maximum Neutron Star Mass. Nucl. Phys. A 2016, 950, 64-109.

10. Riek, F.; Lutz, M.F.M.; Korpa, C.L. Photoabsorption off nuclei with self consistent vertex corrections. Phys. Rev. C 2009, 80, 024902.

11. Ohnishi, A.; Kawamoto, N.; Miura, K. Brown-Rho Scaling in the Strong Coupling Lattice QCD. Mod. Phys. Lett. A 2008, 23, 2459-2464.

12. Brown, G.E.; Rho, M. Double decimation and sliding vacua in the nuclear many body system. Phys. Rept. 2004, 396, 1-39.

13. Paeng, W.G.; Kuo, T.T.S.; Lee, H.K.; Ma, Y.L.; Rho, M. Scale-invariant hidden local symmetry, topology change, and dense baryonic matter. II. Phys. Rev. D 2017, 96, 014031.

14. Van Straaten, S.; Ford, E.C.; van der Klis, M.; Méndez, M.; Kaaret, P. Relations between timing features and colors in the x-ray binary 4u 0614+09. Astrophys. J. 2000, 540, 1049-1061.

15. Ozel, F.; Psaltis, D.; Guver, T.; Baym, G.; Heinke, C.; Guillot, S. The Dense Matter Equation of State from Neutron Star Radius and Mass Measurement. Astrophys. J. 2016, 820, 28. 
16. Suleimanov, V.F.; Poutanen, J.; Nättilä, J.; Kajava, J.J.E.; Revnivtsev, M.G.; Werner, K. The direct cooling tail method for X-ray burst analysis to constrain neutron star masses and radii. Mon. Not. Roy. Astron. Soc. 2017, 466, 906-913.

17. Bogdanov, S. The Nearest Millisecond Pulsar Revisited with XMM-Newton: Improved Mass-Radius Constraints for PSR J0437-4715. Astrophys. J. 2013, 762, 96.

18. Trümper, J.E.; Burwitz, V.; Haberl, F.; Zavlin, V.E. The puzzles of RX J1856.5-3754: Neutron star or quark star? Nucl. Phys. B (Proc. Suppl.) 2004, 132, 560-565.

19. Lattimer, J.M. The nuclear equation of state and neutron star masses. Ann. Rev. Nucl. Part. Sci. 2012, 62, 485-515.

20. Lattimer, J.M.; Steiner, A.W. Neutron Star Masses and Radii from Quiescent Low-Mass X-ray Binaries. Astrophys. J. 2014, 784, 123.

21. Steiner, A.W.; Lattimer, J.M.; Brown, E.F. Neutron Star Radii, Universal Relations, and the Role of Prior Distributions. Eur. Phys. J. A 2016, 52, 18.

22. Maslov, K.A.; Kolomeitsev, E.E.; Voskresensky, D.N. Making a soft relativistic mean-field equation of state stiffer at high density. Phys. Rev. C 2015, 92, 052801.

23. Drago, A.; Lavagno, A.; Pagliara, G.; Pigato, D. Early appearance of Delta isobars in neutron stars. Phys. Rev. C 2014, 90, 065809.

(C) 2017 by the authors. Licensee MDPI, Basel, Switzerland. This article is an open access article distributed under the terms and conditions of the Creative Commons Attribution (CC BY) license (http:/ / creativecommons.org/licenses/by/4.0/). 